\title{
Sensitive detection of RNA microcrystals using SONICC imaging
}

van Beek, Hans; Handing, Kasia; Gualtier, Ellen; Stagno, Jason; Wang, Yun-Xing

The discovery of non-coding RNA has radically changed and expanded the understanding of RNA biology. It has been shown that RNA is not simply an intermediate between genetic information and proteins, but also has catalytic activity and regulates gene expression. The function of RNA is related to its 3D structure and its interaction with proteins, both of which can be studied using X-ray crystallography. Crystallization of RNAs has proven far more challenging than their protein partners. In many cases, obtaining sufficiently-sized crystals for single crystal X-ray diffraction is unlikely. Conversely, much smaller crystals, or nanocrystals (NCs), are often missed due to the limitations of optical methods used for visualization. However, NCs can be a valuable source of structural information given the opportunities offered by X-FEL and micro-focused beam lines in the field of crystallography. Therefore, the development of efficient methods to detect and visualize RNA NCs is important to expand the capabilities in the field of RNA structural biology.

Second Order Nonlinear Imaging of Chiral Crystals (SONICC) has proven to be a sensitive method for the detection of protein NCs less than $1 \mu \mathrm{m}$ in size. Here, we show that SONICC can be used to visualize RNA NCs that are not easily detected with conventional imaging techniques such as visible and UV microscopy. Additionally, we demonstrate that by utilizing Multi Fluorescence Imaging (MFI) one can distinguish between protein and RNA in RNA-protein complexes or between two different proteins in protein-protein complexes. The use of an automated imaging system with SONICC and MFI allows hundreds of crystallization plates to be screened and images viewed to quickly identify positive crystallization conditions for further optimization or x-ray diffraction.

$\begin{array}{ll}\text { Hans van Beek } & \text { Formulatrix } \\ \text { Kasia Handing } & \text { Formulatrix } \\ \text { Ellen Gualtier } & \text { Formulatrix } \\ \text { Jason Stagno } & \text { NIH } \\ \text { Yun-Xing Wang } & \text { NIH } \\ \text { Lance Ramsey } & \text { Formulatrix }\end{array}$

